

\title{
Intramolecular Baylis-Hillman and Morita Reactions Using Unsaturated Thiol Ester Substrates Containing Enolizable Aldehydes
}

\author{
Gary E. Keck and Dennie S. Welch \\ Department of Chemistry, University of Utah, Salt Lake City, Utah 84112-0850 \\ keck@chemistry.utah.edu
}

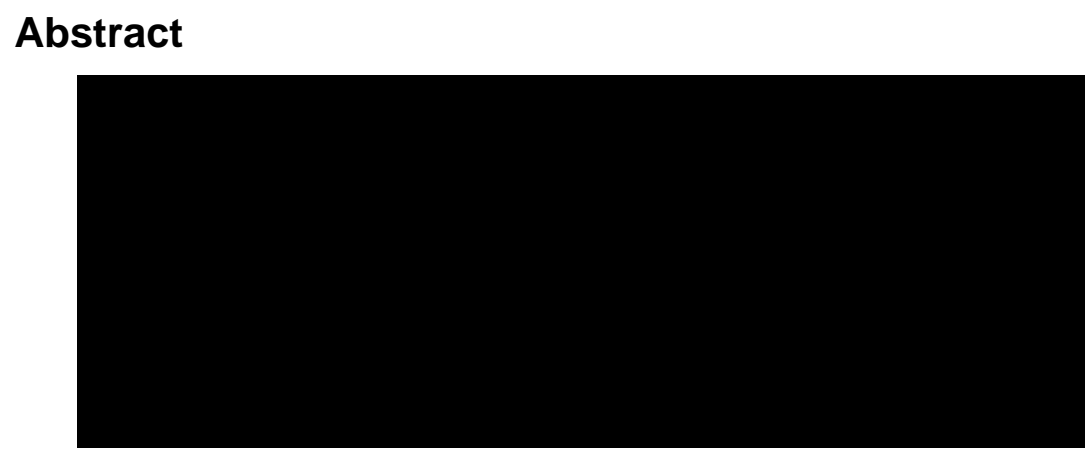

Previously unknown intramolecular Baylis-Hillman and Morita reactions involving cyclization of an unsaturated thiol ester onto a pendant aldehyde function are reported. These can be used successfully for the preparation of both cyclopentenols and cyclohexenols, but the results are very sensitive to substrate and precise reaction conditions.

Recently, in the context of a synthetic project underway in our laboratories, we had need of a functionalized cyclopentenol containing the general structural motif embodied in structure 1 below. In considering potential approaches to the synthesis of such an intermediate, an intramolecular version of the Baylis-Hillman reaction ${ }^{1}$ appeared to be an especially direct possibility (eq 1).

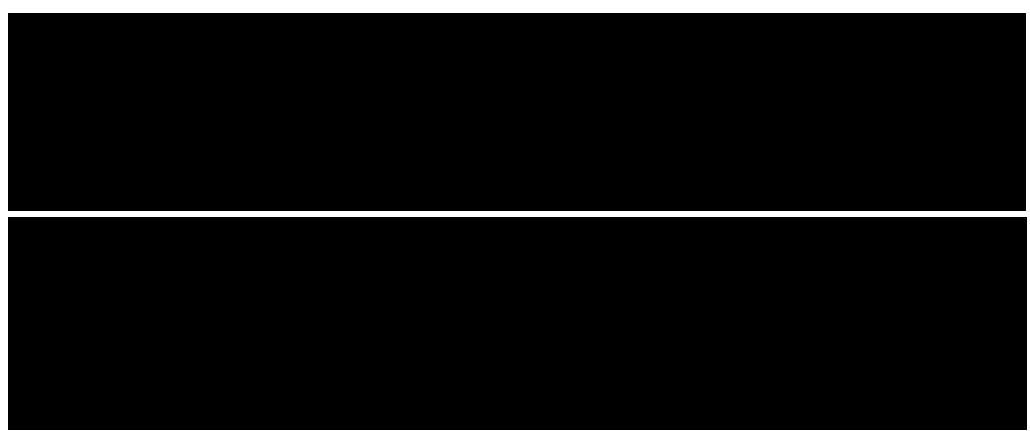

However, attempts to identify precedent for such a process revealed that examples of the intramolecular Baylis-Hillman reaction, although known, are exceedingly rare. The first such report utilized an unsaturated ester tethered to a methyl ketone and was successful using

Supporting Information Available: Spectral and analytical data and experimental procedures. This material is available free of charge via the Internet at http://pubs.acs.org. 
phosphine catalysis. ${ }^{2}$ The only aldehyde-containing substrates for which such a reaction has been reported are for one nonenolizable case that led to a coumarin derivative ${ }^{3}$ and two examples reported by Murphy and co-workers ${ }^{4}$ using an enone-containing substrate bearing an enolizable aldehyde. Even in this latter case where an enone could be used successfully in a reaction promoted by piperidine, the corresponding ethyl ester failed to give any of the desired Baylis-Hillman product. Instead, either no reaction or aldol condensation was observed.

Subsequent studies using tri- $n$-butylphosphine (Morita reaction) in $\mathrm{CHCl}_{3}$ with such enones were successful for six-ring formation but gave poor yields $(20 \%)$ for five-ring formation. ${ }^{5} \mathrm{~A}$ full report details limited success for cyclizations of an enoate or thioenoate onto an enolizable aldehyde. ${ }^{6}$ It has been previously reported that both DBU and tributylphosphine are ineffective in promoting Baylis-Hillman-type reactions of $S$-ethylthioacrylate with aldehydes. ${ }^{7}$

It occurred to us that previous observations from these laboratories could potentially afford a solution to these problems and allow for the synthesis of such cyclopentenols using the intramolecular Baylis-Hillman approach (Figure 1). Specifically, we had previously demonstrated that $Z \alpha, \beta$-unsaturated thiol esters could be easily isomerized to $E$ by exposure to catalytic amounts of DMAP in $\mathrm{CH}_{2} \mathrm{Cl}_{2}$ at room temperature. ${ }^{8}$ Presumably this process proceeds by a conjugate addition- $\beta$ elimination sequence. Normal oxo-esters were unaffected by such treatment.

It thus appeared that DMAP should be competent to serve as a Baylis-Hillman catalyst with appropriate thiol ester substrates. We record herein the results of an investigation into this reaction that provide efficient access to the desired cyclopentenols and offer some further insight into the Baylis-Hillman reaction as well.

Initial attempts to induce cyclization simply by exposure of 3 to DMAP in $\mathrm{CH}_{2} \mathrm{Cl}_{2}$ at room temperature gave no reaction. Since our previous work strongly suggested that the required initial 1,4 addition occurs readily under these conditions, it appeared that the ensuing intramolecular aldol step was too slow to compete with $\beta$-elimination of the pyridyl group. We suspected that some additional activation for the aldehyde carbonyl might be required to allow this step to proceed smoothly. Successful aldol reactions involving enolates generally require transfer of metal ion from enolate oxygen to the developing alkoxide oxygen in the product; otherwise, loss of delocalization energy in going from enolate to alkoxide renders the aldol equilibrium unfavorable. This principle is now well recognized in the context of the bimolecular aldol reaction (Zimmerman-Traxler transition state). ${ }^{9}$ Elegant work by Noyori has also shown that "naked" enolates do not undergo simple aldol condensation reactions. 10

This reaction was therefore investigated in the presence of additives that could potentially mediate the overall reaction by providing stabilization for the aldol portion of the pathway. Conditions under which reaction via an enol rather than enolate ion could reasonably be expected were also examined; these likewise allow for a low energy pathway to product. The results of a number of these experiments are summarized in Table 1.

Attempts to force reaction by employing elevated temperatures with DMAP alone were marginally successful; thus, raising the temperature to $65{ }^{\circ} \mathrm{C}$ (reaction in $\mathrm{CHCl}_{3}$ ) gave a $50 \%$ yield of the desired Baylis-Hillman product 4 after $48 \mathrm{~h}$. Addition of DMAP•HCl to the $\mathrm{CH}_{2} \mathrm{Cl}_{2}$ room-temperature reaction was without effect; however, with the addition of DMAP $\cdot \mathrm{HCl}$, reaction did occur more rapidly in $\mathrm{CHCl}_{3}$ at $65^{\circ} \mathrm{C}$, to afford 4 in $48 \%$ yield after $24 \mathrm{~h}$ (entry 4). Other solvents were then explored in an attempt to improve upon these results. No reaction was observed using DMAP and DMAP• $\mathrm{HCl}$ in THF at $65^{\circ} \mathrm{C}$ or in DMF at $23^{\circ}$ C. Reaction was observed in DMF at higher temperatures, but the yield ( $43 \%$ after $5 \mathrm{~h}$ at $78^{\circ}$ C, entry 8) was still low. 
Much better results were obtained using protic solvents. ${ }^{11}$ Although no reaction was observed in EtOH using conditions that mimicked those of the previously reported $Z-E$ isomerization (catalytic amounts of DMAP at room temperature) use of a full equivalent of DMAP in EtOH at $78{ }^{\circ} \mathrm{C}$ afforded the desired 4 in $72 \%$ yield after only $2.5 \mathrm{~h}$. Inclusion of DMAP•HCl gave a much cleaner reaction (TLC analysis) and improved the yields (entry 11). Optimal results in terms of yield were observed with 1.0 equiv of DMAP and 0.25 equiv of DMAP $\cdot \mathrm{HCl}$, in which case an $87 \%$ isolated yield was obtained after 1 hat $78^{\circ} \mathrm{C}$ (entry 12). Comparison with entry 11 shows that although lesser quantities of reagent can be employed, reaction times are correspondingly longer. Moreover, this ratio of DMAP to DMAP• $\mathrm{HCl}$ was empirically determined to be optimal for this reaction. For example, as shown in entry 13, using a full equivalent of DMAP• $\mathrm{HCl}$ under otherwise identical conditions lowers the yield to $60 \%$ at the same reaction time.

Having identified conditions under which the desired reaction was high yielding, it was of considerable interest to compare these results with those obtained using amines more typically employed in the Baylis-Hillman process. ${ }^{12,13}$ Treatment of $\mathbf{3}$ with DBU in $\mathrm{CH}_{2} \mathrm{Cl}_{2}$ led to complete consumption of starting material within $3 \mathrm{~h}$; however, 4 could be isolated in only $25 \%$ yield. Reactions were also conducted in EtOH using both DABCO and DBU to allow for direct comparison with the DMAP results in the same solvent. With DABCO, although the starting material was completely consumed within $1 \mathrm{~h}$ at $78{ }^{\circ} \mathrm{C}$, the desired 4 was obtained in only $18 \%$ yield. The major product obtained in this case was the acetal $\mathbf{5}$. With $D B U$, this process was much more facile, and 5 was obtained quantitatively within 5 min at $23^{\circ} \mathrm{C}$. Thus, these more basic amines cannot be used in the protic media in which the Baylis-Hillman process is intrinsically more favorable, as a result of the intrusion of this alternative reaction pathway.

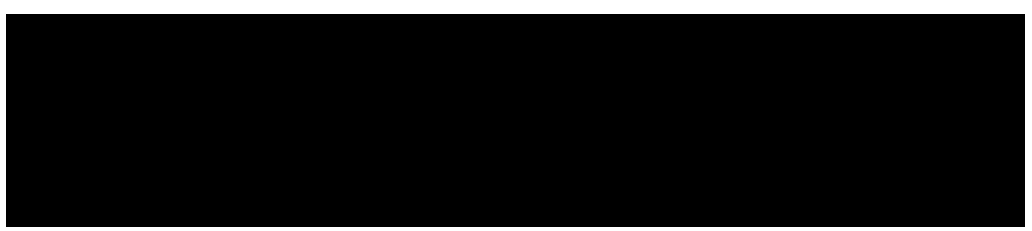

Attempts to use added lithium ion $\left(\mathrm{LiCl}\right.$ or $\left.\mathrm{LiClO}_{4}\right)$ in THF or ether to promote the DMAPmediated reaction were unsuccessful. However, the use of THF-water as solvent with DMAP $\cdot \mathrm{HCl}$ present gave good results. In this case, a $63 \%$ yield could be obtained in a roomtemperature reaction. Conducting the same reaction at $65^{\circ} \mathrm{C}$ afforded the desired product in $70 \%$ isolated yield after $30 \mathrm{~min}$. Although these reactions in aqueous THF were quite fast, the isolated yields were never quite as high as those obtained in the EtOH reactions.

Several other potential substrates for this reaction were prepared to assess the impact of substitution patterns upon the viability of this approach to constructing such cyclopentenols. The results of these reactions are summarized in Figure 2, as are the results obtained using $\mathrm{PMe}_{3}$ as catalyst (vide infra). Gem dimethyl substitution adjacent to the aldehyde carbonyl was very well tolerated; thus cyclopentenol 6 could be prepared in $88 \%$ yield using the reaction conditions optimized for 3 . Steric hindrance adjacent to the $\beta$-carbon of the unsaturated thiol ester was less well tolerated and encouraged reaction via initial hemiacetal formation.

Cyclopentenol 7 was obtained in $41 \%$ yield from the corresponding aldehyde substrate, along with $58 \%$ of the ethoxy tetrahydrofuran product.

Curiously, attempts to extend this reaction to the preparation of a cyclohexenol gave much lower yields. Application of the protocol that proved optimal with $\mathbf{3}$ to the otherwise identical six-ring case afforded the desired product 8 in only $29 \%$ yield after $48 \mathrm{~h}$ at reflux. The oxoester corresponding to $\mathbf{3}$ also proved, as expected, to be much less reactive, affording only a $40 \%$ yield of cyclopentenol product 2 after $48 \mathrm{~h}$ at reflux. 
After these studies had been completed, two independent reports appeared on intramolecular versions of the very closely related vinylogous Baylis-Hillman-Morita reaction, both using phosphine catalysis. Krische ${ }^{14}$ reported a number of enone-enone cyclization reactions, while Roush ${ }^{15}$ reported enone-enal, enal-enal, and enal-enoate cyclizations, as shown in Figure $3 .{ }^{16}$ Although the one previous report of the use of phosphine catalysis in an intramolecular Baylis-Hillman reaction was not encouraging, it was clearly of interest to examine the protocols developed by Roush and by Krische with our thiol ester-aldehyde substrates.

The conditions that were generally optimal in the Roush study were most thoroughly investigated. Reaction of the substrate 3 with trimethylphosphine ( 0.2 equiv) in tert-amyl alcohol $(0.1 \mathrm{M}$ in 3$)$ at room temperature for $3 \mathrm{~h}$ afforded none of the desired Baylis-Hillman adduct, although starting material was consumed with the formation of numerous unidentified materials. However, in $\mathrm{CH}_{2} \mathrm{Cl}_{2}$ at room temperature, reaction with trimethylphosphine (1.0 equiv) was complete in $0.5 \mathrm{~h}$ and gave 4 in $56 \%$ isolated yield. Further experimentation showed that this reaction gave higher yields at lower substrate concentration and lower catalyst loading. Under optimal conditions, an $82 \%$ yield of 4 was obtained after $15 \mathrm{~h}$ when the reaction was conducted in $\mathrm{CH}_{2} \mathrm{Cl}_{2}$ at room temperature, $0.02 \mathrm{M}$ in substrate, with 0.1 equiv of $\mathrm{PMe}_{3}$.

Given the success of this reaction, we were curious to determine whether this protocol could be applied to the problematic case of six-ring formation. Reaction of the thiol ester substrate under these conditions led to the desired cyclohexenol 8 in 56\% yield. Further experimentation showed that with this substrate the yield could be improved by slightly higher catalyst loading relative to that of the parent case. The best results for this case were obtained using 0.25 equiv of trimethylphosphine in $\mathrm{CH}_{2} \mathrm{Cl}_{2}$ at $0.02 \mathrm{M}$ in substrate, which afforded a $75 \%$ isolated yield of 8 after $2 \mathrm{~h}$ at room temperature. In view of the excellent results obtained using this procedure, it was applied to the other materials studied using the DMAP conditions. A comparison of the results obtained using the DMAP and $\mathrm{PMe}_{3}$ protocols is summarized in Figure 2. The results depicted are for the conditions that proved optimal for each substrate.

In summary, two protocols for the intramolecular Baylis-Hillman-Morita reaction have been developed that allow for the use of alkenes activated by substituents at the ester oxidation state. Under the conditions reported, side reactions that complicate the use of enolizable aldehydes, such as bimolecular condensations, are minimized. The results point out the extreme sensitivity of such reactions to small variations in experimental conditions. The availability of an alternative reaction pathway initiated by hemiacetal formation with these aldehyde containing substrates provides another complicating aspect. These observations suggest that negative results in such reactions be viewed with some suspicion unless it is clear that the relevant reaction variables have been thoroughly examined.

\section{Supplementary Material}

Refer to Web version on PubMed Central for supplementary material.

\section{Acknowledgment}

Financial support from the National Institutes of Health (through GM-28961) is gratefully acknowledged.

\section{References}

1. (a) Drewes SE, Roos GHP. Tetrahedron 1988;44:4653.For reviews, see: (b) Basavaiah D, Rao PD, Hyma RS. Tetrahedron 1996;52:8001.

2. Roth F, Gygax P, Frater G. Tetrahedron Lett 1992;33:1045.

3. Drewes SE, Njamela OL, Emslie ND, Ramesar N, Field JS. Synth. Commun 1993;23:2807. 
4. Black GP, Dinon F, Fratucello S, Murphy PJ, Nielsen M, Williams HL, Walshe NDA. Tetrahedron Lett 1997;38:8561.

5. Dinon F, Richards E, Murphy PJ, Hibbs DE, Hursthouse MB, Malik KMA. Tetrahedron Lett 1999;40:3279.

6. Richards EL, Murphy PJ, Dinon F, Fratucello S, Brown PM, Gelbrich T, Hursthouse MB. Tetrahedron 2001;57:7771.

7. Kataoka T, Iwama T, Kinoshita H, Tsujiyama S, Tsurukami Y, Iwamura T, Watanabe S. Synlett 1999:197.

8. Keck GE, Boden EP, Mabury SA. J. Org. Chem 1985;50:709.

9. Zimmerman HE, Traxler MD. J. Am. Chem. Soc 1957;79:1920.

10. Noyori R, Nishida I, Sakata J. J. Am. Chem. Soc 1983;105:1598.

11. (a) Aggarwal VK, Dean DK, Mereu A, Williams R. J. Org. Chem 2002;67:510. [PubMed: 11798324] For rate accelerations of bimolecular Baylis-Hillman reactions in protic media, see: (b) Yu C, Liu B, Hu L. J. Org. Chem 2001;66:5413. [PubMed: 11485463]

12. Rezgui F, El Gaied MM. Tetrahedron Lett 1998;39:5965.DMAP has been used in the Baylis-Hillman reaction of cyclohexenone with aqueous formaldehyde:

13. Aggarwal VK, Mereu A. Chem. Commun 1999:2311.It has been suggested that DBU is the best amine promoter for the Baylis-Hillman reaction:

14. Wang L-C, Luis AL, Agapiou K, Jang H-Y, Krische MJ. J. Am. Chem. Soc 2002;124:2402. [PubMed: 11890765]

15. Frank SA, Mergott DJ, Roush WR. J. Am. Chem. Soc 2002;124:2404. [PubMed: 11890766]

16. Neither author reported cyclizations in which the initial nucleophilic addition occurred to an enoate or thioenoate. 


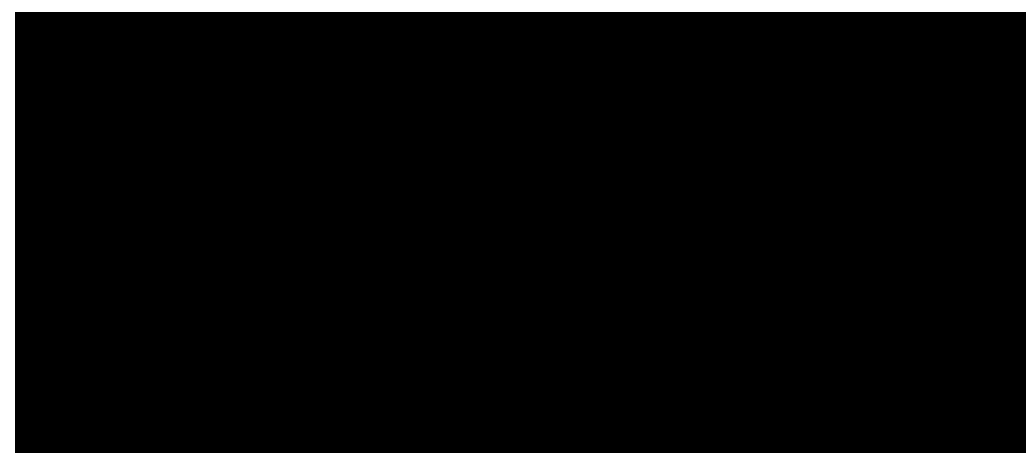

Figure 1.

Previous observation and hypothesis. 


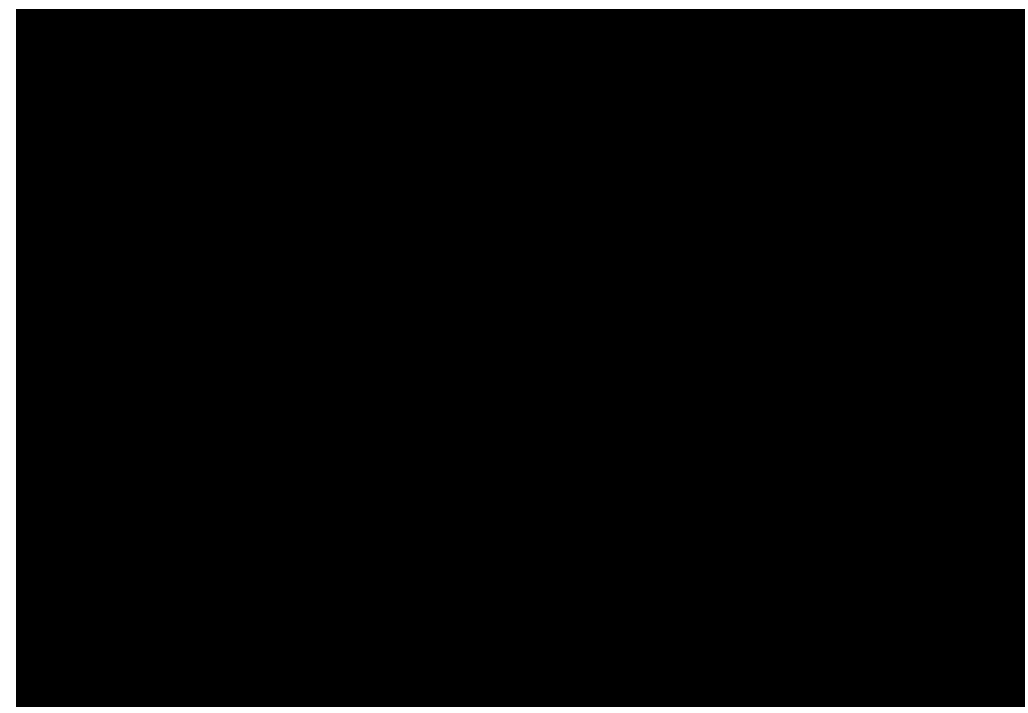

Figure 2.

Isolated yields for Baylis-Hillman-Morita cyclizations using DMAP/DMAP•HCl/EtOH or $\mathrm{PMe}_{3} / \mathrm{CH}_{2} \mathrm{Cl}_{2}$. 




Figure 3.

Vinylogous cyclizations by Krische and Roush. 
Table 1

Reaction Conditions and Isolated Yields for Baylis-Hillman Cyclization of $\mathbf{3}$ with Amine and Phosphine Promoters

\begin{tabular}{|c|c|c|c|c|c|c|}
\hline entry & solvent & temp $\left({ }^{\circ} \mathrm{C}\right)$ & time & additive & equiv & yield $(\%)^{a}$ \\
\hline 1 & $\mathrm{CH}_{2} \mathrm{Cl}_{2}$ & $\mathrm{rt}$ & $\sim 24 \mathrm{~h}$ & DMAP & 1.0 & no reaction \\
\hline 2 & $\mathrm{CHCl}_{3}$ & 65 & $48 \mathrm{~h}$ & DMAP & 1.0 & $50^{b}$ \\
\hline 3 & $\mathrm{CH}_{2} \mathrm{Cl}_{2}$ & $\mathrm{rt}$ & $\sim 24 \mathrm{~h}$ & DMAP, DMAP.HCl & $0.5,0.3$ & no reaction \\
\hline 4 & $\mathrm{CHCl}_{3}$ & 65 & $\sim 24 \mathrm{~h}$ & DMAP, DMAP.HCl & $1.0,0.25$ & $48^{c}$ \\
\hline 5 & $\mathrm{CH}_{2} \mathrm{Cl}_{2}$ & $\mathrm{rt}$ & $3 \mathrm{~h}$ & DBU & 1.0 & 25 \\
\hline 6 & $\mathrm{THF}$ & 65 & $10 \mathrm{~h}$ & DMAP, DMAP.HCl & $1.0,0.25$ & no reaction \\
\hline 7 & $\mathrm{DMF}$ & $\mathrm{rt}$ & $3 \mathrm{~h}$ & DMAP, DMAP. $\mathrm{HCl}$ & $1.0,0.25$ & no reaction \\
\hline 8 & DMF & 78 & $5 \mathrm{~h}$ & DMAP. DMAP.HCl & $1.0,0.25$ & 43 \\
\hline 9 & EtOH & $\mathrm{rt}$ & $45 \mathrm{~h}$ & DMAP & 0.1 & no reaction \\
\hline 10 & $\mathrm{EtOH}$ & 78 & $2.5 \mathrm{~h}$ & DMAP & 1.0 & 72 \\
\hline 11 & $\mathrm{EtOH}$ & 78 & $2 \mathrm{~h}$ & DMAP, DMAP. $\mathrm{HCl}$ & $0.5,0.125$ & 84 \\
\hline 12 & EtOH & 78 & $1 \mathrm{~h}$ & DMAP, DMAP.HCI & $1.0,0.25$ & 87 \\
\hline 13 & $\mathrm{EtOH}$ & 78 & $1 \mathrm{~h}$ & DMAP, DMAP.HCl & $1.0,1.0$ & 60 \\
\hline 14 & $\mathrm{EtOH}$ & 78 & $1 \mathrm{~h}$ & $\mathrm{DABCO}$ & 1.0 & 18 \\
\hline 15 & EtOH & $\mathrm{rt}$ & $5 \mathrm{~min}$ & DBU & $\begin{array}{l}1.0 \\
1.0\end{array}$ & $0^{10} d$ \\
\hline 16 & $\begin{array}{l}\mathrm{THF} / \mathrm{H}_{2} \mathrm{O} \\
(1: 1)\end{array}$ & 65 & $0.5 \mathrm{~h}$ & DMAP, DMAP.HCl & $1.0,0.25$ & 70 \\
\hline 17 & $\begin{array}{l}\mathrm{THF} / \mathrm{H}_{2} \mathrm{O} \\
(4: 1)\end{array}$ & $\mathrm{rt}$ & $4 \mathrm{~h}$ & DMAP, DMAP.HCl & $1.0,0.25$ & 63 \\
\hline 18 & $\begin{array}{l}\text { tert-amyl } \\
\text { alcohol }\end{array}$ & $\mathrm{rt}$ & $3 \mathrm{~h}$ & $\mathrm{PMe}_{3}$ & 1.0 & 0 \\
\hline 19 & $\mathrm{CH}_{2} \mathrm{Cl}_{2}$ & $\mathrm{rt}$ & $0.5 \mathrm{~h}$ & $\mathrm{PMe}_{3}$ & 1.0 & 56 \\
\hline 20 & $\mathrm{CH}_{2} \mathrm{Cl}_{2}$ & $\mathrm{rt}$ & $15 \mathrm{~h}$ & $\mathrm{PMe}_{3}$ & 0.1 & 82 \\
\hline
\end{tabular}

${ }^{a}$ Unless otherwise indicated, complete consumption of starting material was observed and substrate concentration was $0.1 \mathrm{M}$.

$b_{46 \%}$ of starting material was recovered.

$c_{18 \%}$ of starting material was recovered.

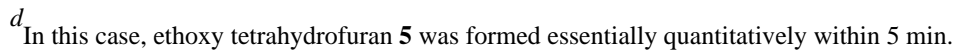

